\title{
Mycobacterium chimaera colonisation of heater-cooler units (HCU) in Western Australia, 2015: investigation of possible iatrogenic infection using whole genome sequencing
}

\author{
JO Robinson ${ }^{1234}$, GW Coombs ${ }^{34}$, DJ Speers ${ }^{56}$, T Keehner 5 , AD Keil 5 , V D’Abrera 7 , P Boan ${ }^{23}$, S Pang ${ }^{34}$ \\ 1. Royal Perth Hospital, Perth, Australia \\ 2. Fiona Stanley Hospital, Perth Australia \\ 3. Pathwest Laboratory Medicine WA, Fiona Stanley Hospital Network, Perth, Australia \\ 4. Australian Collaborating Centre for Enterococcus and Staphylococcus Species (ACCESS) Typing and Research, School of \\ Veterinary and Life Sciences, Murdoch University and School of Biomedical Sciences, Curtin University, Perth, Australia \\ 5. PathWest Laboratory Medicine WA, Hospital Avenue, Nedlands, Australia \\ 6. School of Medicine and Pharmacology, University of Western Australia, Crawley, Australia \\ 7. St John of God Pathology, Perth, Australia
}

Correspondence: James Owen Robinson (owen.robinson@health.wa.gov.au)

Following the reported link between heater-cooler unit (HCU) colonisation with Mycobacterium chimaera and endocarditis, mycobacterial sampling of all HCUs in use in Western Australia was initiated from August 2015, revealing $M$. chimaera colonisation in 10 of 15 HCUs. After M. chimaera was isolated from a pleural biopsy from a cardiothoracic patient who may have been exposed to a colonised HCU, a whole genome sequencing investigation was performed involving 65 specimens from $15 \mathrm{HCUs}$ across five hospitals to assess if this infection was related to the HCU. Genetic relatedness was found between the $10 \mathrm{HCU} M$. chimaera isolates from four hospitals. However the $M$. chimaera isolate from the cardiothoracic patient was not genetically related to the HCU M. chimaera isolates from that hospital, nor to the other HCU isolates, indicating that the HCUs were not the source of the infection in this patient.

\section{Introduction}

Mycobacterium chimaera is a slow growing mycobacterium from the Mycobacterium avium complex. In 2004, $M$. chimaera was identified as a new species within the complex group [1] and has been associated with pulmonary infections, predominantly in immunosuppressed patients and in patients with pre-existing lung conditions such as chronic obstructive pulmonary disease and cystic fibrosis [2,3]. Since 2013, M. chimaera has been reported as a cause of prosthetic valve endocarditis, bloodstream and vascular graft infections in several countries in Europe and the United States (US) [4-10], linked to the colonisation of the heater-cooler units (HCUs) used during open heart surgery with postulated airborne transmission in the operating theatre $[8,11]$.

In June 2015, an HCU manufacturer issued a warning, instructing hospitals to follow updated disinfection and maintenance procedures on HCUs and perform mycobacterial sampling. In response, private and public hospitals in Western Australia (WA) commenced mycobacterial sampling of their HCUs (all HCUs in WA have been purchased from the same manufacturer) in August 2015. In March 2016, a clinical isolate of $M$. chimaera was obtained from a cardiothoracic surgical patient in whom the surgery involved the use of one of the HCUs. This finding triggered an investigation to assess if this infection was related to the HCU.

\section{Case report and environmental sampling}

A patient in their 50 s underwent cardiothoracic surgery employing a HCU for cardiopulmonary bypass at Hospital 4 in December 2015. The surgery did not involve the implantation of prosthetic material. During this surgery, the HCU was placed as far as possible from the patient, with the exhaust towards the theatre exhaust vent and away from the patient.

Following the warning from the manufacturer, the water of all five HCUs at Hospital 4 was cultured and four of the five HCUs tested positive for M. chimaera in October 2015. All HCUs therefore underwent cleaning and disinfection following the manufacturer's instruction and were then deemed safe for use, the risk of 


\section{FIGURE}

Genomic analysis of Mycobacterium chimaera strains grown from heater-cooler units from four hospitals, the cardiothoracic patient from Hospital 4 and a noncardiothoracic patient, Western Australia, 2015-16 ( $\mathrm{n}=12)$
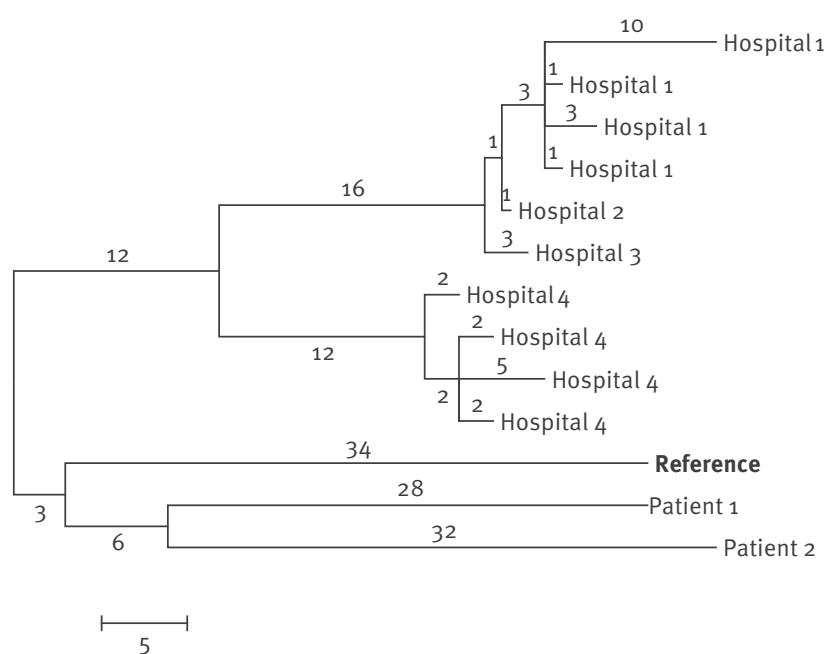

Patient 1: Hospital 4 cardiothoracic patient isolate, December 2015; patient 2: non-cardiothoracic patient isolate; reference: M chimaera strain MCIMRL6 (NCBI accession number: LJHN01000001)

Numbers indicate single nucleotide polymorphism differences.

postponing surgery while waiting for culture results being greater than the potential residual infective risk. As part of the monthly testing protocol, the HCUs were again sampled in November 2015 and M. chimaera was again cultured from one of the five HCUs after a 53-day incubation, i.e. after the patient's surgery. As the specific HCU used at the time of surgery was not recorded, it was not possible to conclude or exclude that the patient was exposed to a HCU colonised with $M$. chimaera. A process of recording the HCU used for each patient surgery has since been introduced.

Air sampling was also attempted from the operating theatres at Hospital 4, but the sampling plates were overgrown with other organisms such that interpretation of mycobacterial growth was not possible. Sampling from other hospital sources, such as potable water was not attempted.

One month after the operation, the patient developed bilateral pleural effusions and a pneumothorax with Pseudomonas aeruginosa isolated from the pleural fluid. During a 6-week course of piperacillin/ tazobactam, the patient required four pleural drainage procedures, three for recurrent effusion and one for pneumothorax. One week after cessation of antibiotics, the patient redeveloped a pleural effusion and $P$. aeruginosa was again cultured. At this point the patient underwent decortication, and $M$. chimaera was cultured from a pleural biopsy. The patient was commenced on a combination of piperacillin/tazobactam, ciprofloxacin, azithromycin and ethambutol and slowly improved. Of note, the patient did not have signs and symptoms of disseminated $M$. chimaera infection. Mycobacterial blood cultures were not performed.

\section{Methods}

Mycobacterial culture from HCUs was performed at the Western Australian mycobacterial reference laboratory. Mycobacteriology culture methods for water samples based on the 2010 Gastroenterological Society of Australia guidelines [12] and comparable with subsequent British [13] and European [14] guidelines for $M$. chimaera isolation were followed. Aliquots of $50 \mathrm{~mL}$ were centrifuged at 3,000 $\mathrm{g}$ for $20 \mathrm{~min}$, the supernatant discarded and the remaining 1-2 $\mathrm{mL}$ decontaminated using n-acetyl-l-cysteine-sodium hydroxide/ sodium citrate. Two BBL MGIT tubes (Mycobacteria Growth Indicator Tube, Becton Dickinson, Sparks, US) and two Gerloff's egg slopes (with added nalidixic acid, vancomycin, amphotericin and polymyxin) were each inoculated with $0.5 \mathrm{~mL}$ of the processed sample and incubated for 8 weeks at $30^{\circ} \mathrm{C}$ and $36^{\circ} \mathrm{C}$. Positive cultures were confirmed by acid fast staining, with subculturing on Middlebrook $7 \mathrm{H}_{11}$ plates for purity and identification. Single colony identification was performed by $16 \mathrm{~S}$ rRNA gene sequencing.

The pleural biopsy was similarly cultured but without $\mathrm{NaOH}$ processing, with the clinical isolate initially identified on solid media at $30^{\circ} \mathrm{C}$ after 21 days.

Whole genome sequencing (WGS), using aMiSeq platform (Illumina, San Diego, US), was performed on all HCU M. chimaera isolates, the patient isolate and a $M$. chimaera isolate from a non-cardiothoracic patient. M. chimaera strain MCIMRL6 (NCBI accession number: LJHN01000001), a clinical respiratory isolate, was used as the reference sequence [15]. The Illumina pairedend sequencing data, with an average of $70 \times$ coverage depth, were analysed for genetic relatedness using the Nullarbor bioinformatic pipeline software [16] to identify single nucleotide polymorphisms (SNPs) in the core genome by comparison with the reference sequence. SNPs in recombination events were removed based on the method described by Feng et al. A maximum parsimony phylogenetic tree was constructed using MEGA (v7.0) [17].

\section{Results}

Sixty-five specimens from 15 HCUs used in five WA hospitals were cultured for mycobacteria over a 12-month period from August 2015 to July 2016. The sampling pattern initially varied between hospitals but became more regular for all hospitals with HCUs over time as standardised testing intervals were established. Single mycobacterial isolates from 10 different HCUs from four hospitals, as well as the patient isolate and the second clinical isolate from a non-cardiothoracic patient were confirmed as $M$. chimaera by WGS. In addition, $M$. intracellulare and $M$. gordonae were also isolated from HCUs. The M. chimaera HCU isolates clustered into two groups, one from Hospital 4 and one from Hospitals 
1-3. The two groups differed by 28 SNPs, with $2-17$ SNP differences between isolates within a group. The isolate from the patient in Hospital 4 did not cluster with the Hospital $4 \mathrm{HCU}$ isolates; it differed from them by at least 63 SNPs (Figure). Likewise, the non-cardiothoracic patient isolate did not cluster with the HCU isolates.

\section{Discussion}

An association of HCU colonisation with $M$. chimaera and subsequent deep tissue infections in cardio-pulmonary bypass patients has been reported in Europe and the US $[5-7,11]$ but published molecular epidemiological information is scarce [8]. Our WGS investigation revealed frequent $M$. chimaera colonisation of HCUs across several hospitals in WA. The WGS results show that the HCU M. chimaera isolates in WA were genetically related as they all shared common SNPs, which is consistent with contamination from a common source. There was no transfer of HCUs between hospitals in WA to implicate a single hospital contamination event. The hospital tap water supply cannot be excluded as a source, but only sterile packaged water or filtered tap water is used in the filling and cleaning of HCUs in WA. Given that all the HCUs in WA were produced at the same manufacturing site, one hypothesis is the HCUs were contaminated during production at this site, as recently suggested by Haller et al. [6]. In their study they showed that isolates from cardiothoracic patients, HCUs and the manufacturing site were almost identical; however, their typing results have not been published. To examine this hypothesis further, systematic WGS of isolates collected from HCUs in multiple geographical locations is required. Notably, the two HCUs from the WA hospital that did not yield any positive cultures for $M$. chimaera were significantly older (more than 10 years) than the HCUs in Hospitals 1-4 and thus may have been manufactured before a possible contamination event at the manufacturing site.

The $M$. chimaera isolate from the cardiothoracic patient was not genetically related to the HCU M. chimaera isolates from that hospital, nor to the other HCU isolates, indicating that the HCUs were not the source of the infection in this patient. Although this finding is reassuring, the presence of multiple different strains in an individual specimen may not have been detected by our sampling method as only one colony was selected from the culture media for WGS. Furthermore, $M$. chimaera cases have been diagnosed up to five years after cardiovascular surgery [6] and therefore we may detect linked clinical cases into the future.

Interestingly, both patient isolates and the reference strain were from respiratory specimens but were not closely related to each other or to the HCU isolates. This would suggest heterogeneity in the environmental $M$. chimaera populations able to infect the respiratory tract of these patients and the HCUs. Due to the probability of contamination with $M$. chimaera at the overseas manufacturing site it is possible that the observed genetic differences between the patient and $\mathrm{HCU}$ isolates may simply reflect different $M$. chimaera populations in water sources in the two countries. It is currently unknown if different $M$. chimaera strains have different pathogenicity to cause infections of either prosthetic heart valves or the respiratory tract.

\section{Conclusion}

Our study has demonstrated the usefulness of WGS in the analysis of a potential iatrogenic $M$. chimaera infection and shown that some HCUs used in WA are colonised with $M$. chimaera, as observed in countries on the northern hemisphere. As yet, no HCU-related infections have been identified in patients undergoing cardiopulmonary bypass procedures in WA. We must maintain a high level of suspicion in the population at risk while continuing regular disinfection and mycobacterial monitoring of our HCUs.

\section{Acknowledgements}

The authors wish to acknowledge the hospital medical perfusionists for collection of the HCU samples.

\section{Conflict of interest}

None declared.

Authors' contributions

James Owen Robinson: initiated the research, analysed the data and wrote the manuscript.

Stanley Pang: generated the whole-genome sequencing data, analysed the data, produced the figure and reviewed the manuscript.

David John Speers, Terillee Keehner, Anthony David Keil, Victoria D’Abrera, Peter Boan, Geoffrey Wallace Coombs: analysed the data and reviewed the manuscript.

\section{References}

1. Tortoli E, Rindi L, Garcia MJ, Chiaradonna P, Dei R, Garzelli C, et al. Proposal to elevate the genetic variant MAC-A, included in the Mycobacterium avium complex, to species rank as Mycobacterium chimaera sp. nov. Int J Syst Evol Microbiol. 2004;54(Pt 4):1277-85. DOI: 10.1099/ijs.0.02777-0 PMID: 15280303

2. Boyle DP, Zembower TR, Reddy S, Qi C. Comparison of Clinical Features, Virulence, and Relapse among Mycobacterium avium Complex Species.Am J Respir Crit Care Med. 2015;191(11):13107. DOI: 10.1164/rccm.201501-00670C PMID: 25835090

3. Cohen-Bacrie S, David M, Stremler N, Dubus JC, Rolain JM, Drancourt M. Mycobacterium chimaera pulmonary infection complicating cystic fibrosis: a case report.J Med Case Reports. 2011;5(1):473. DOI: 10.1186/1752-1947-5-473 PMID: 21939536

4. Achermann Y, Rössle M, Hoffmann M, Deggim V, Kuster S, Zimmermann DR, et al. Prosthetic valve endocarditis and bloodstream infection due to Mycobacterium chimaera. J Clin Microbiol. 2013;51(6):1769-73. DOI: 10.1128/JCM.00435-13 PMID: 23536407

5. Götting T, Klassen S, Jonas D, Benk Ch, Serr A, Wagner D, et al. Heater-cooler units: contamination of crucial devices in cardiothoracic surgery. J Hosp Infect. 2016;93(3):223-8. DOI: 10.1016/j.jhin.2016.02.006 PMID: 27101883

6. Haller S, Höller C, Jacobshagen A, Hamouda O, Abu Sin M, Monnet DL, et al. Contamination during production of heatercooler units by Mycobacterium chimaera potential cause for invasive cardiovascular infections: results of an outbreak 
investigation in Germany, April 2015 to February 2016.

Euro Surveill. 2016;21(17):30215. DOI: 10.2807/1560-7917.

ES.2016.21.17.30215 PMID: 27168588

7. Kohler P, Kuster SP, Bloemberg G, Schulthess B, Frank M, Tanner FC, et al. Healthcare-associated prosthetic heart valve, aortic vascular graft, and disseminated Mycobacterium chimaera infections subsequent to open heart surgery. Eur Heart J. 2015;36(40):2745-53. DOI: 10.1093/eurheartj/ehv342 PMID: 26188001

8. Sax H, Bloemberg G, Hasse B, Sommerstein R, Kohler P, Achermann Y, et al. Prolonged Outbreak of Mycobacterium chimaera Infection After Open-Chest Heart Surgery. Clin Infect Dis. 2015;61(1):67-75. DOI: 10.1093/cid/civ198 PMID: 25761866

9. Perkins KM, Lawsin A, Hasan NA, Strong M, Halpin AL, Rodger $\mathrm{RR}$, et al. Notes from the Field: Mycobacterium chimaera Contamination of Heater-Cooler Devices Used in Cardiac Surgery - United States. MMWR Morb Mortal Wkly Rep. 2016;65(40):1117-8. DOI: 10.15585/mmwr.mm6540a6 PMID: 27740609

10. Tan N, Sampath R, Abu Saleh OM, Tweet MS, Jevremovic D, Alniemi S, et al. Disseminated Mycobacterium chimaera Infection After Cardiothoracic Surgery. Open Forum Infect Dis. 2016;3(3):ofw131. DOI: 10.1093/ofid/ofw131 PMID: 27703994

11. Sommerstein R, Rüegg C, Kohler P, Bloemberg G, Kuster SP, Sax H. Transmission of Mycobacterium chimaera from HeaterCooler Units during Cardiac Surgery despite an Ultraclean Air Ventilation System.Emerg Infect Dis. 2016;22(6):1008-13. DOI: 10.3201/eid2206.160045 PMID: 27070958

12. Taylor A, Jones D, Everts R, Cowen A, Wardle E, editors. Infection control in endoscopy. 3 rd ed. Mulgrave: Gastroenterological Society of Australia; 2010. Available from: http://membes.gesa.org.au/membes/files/Clinical\%20 Guidelines\%20and\%20Updates/Infection_Control_in_ Endoscopy_Guidelines_2014.pdf

13. Public Health England (PHE). Protocol for environmental sampling, processing and culturing of water and air samples for the isolation of slow-growing mycobacteria. Standard operating procedure. London: PHE; 2015 Available from: https://www.gov.uk/government/ uploads/system/uploads/attachment_data/file/540325/ Air_water_environmental_sampling_SOP_V2.pdf

14. European Centre for Disease Prevention and Control (ECDC). EU protocol for case detection, laboratory diagnosis and environmental testing of Mycobacterium chimaera infections potentially associated with heater-cooler units: case definition and environmental testing methodology. Stockholm: ECDC; 2015. Available from: http://ecdc.europa.eu/en/publications/ Publications/EU-protocol-for-M-chimaera.pdf

15. Mac Aogáin M, Roycroft E, Raftery P, Mok S, Fitzgibbon M, Rogers TR. Draft Genome Sequences of Three Mycobacterium chimaera Respiratory Isolates.Genome Announc. 2015;3(6):e01409-15. DOI: 10.1128/genomeA.01409-15 PMID: 26634757

16. Seemann T, Goncalves da Silva A, Bulach DM, Schultz MB, Kwong JC, Howden BP. Nullarbor. San Francisco; Github. [Accessed: 03 Jun 2016]. Available from: https://github.com/ tseemann/nullarbor

17. Kumar S, Stecher G, Tamura K. MEGA7: Molecular Evolutionary Genetics Analysis Version 7.0 for Bigger Datasets.Mol Biol Evol. 2016;33(7):1870-4. DOI: 10.1093/molbev/msw054 PMID: 27004904

\section{License and copyright}

This is an open-access article distributed under the terms of the Creative Commons Attribution (CC BY 4.0) Licence. You may share and adapt the material, but must give appropriate credit to the source, provide a link to the licence, and indicate if changes were made.

This article is copyright of the authors, 2016. 